\author{
Khaldoun Koujok \\ Robert E. Ruiz \\ Ramiro J. Hernandez
}

\section{Myofibromatosis: imaging characteristics}

Received: 23 July 2004

Revised: 15 September 2004

Accepted: 23 September 2004

Published online: 19 November 2004

(C) Springer-Verlag 2004
K. Koujok · R. J. Hernandez $(\bowtie)$

Department of Radiology, CS Mott Children's Hospital,

University of Michigan Health System,

1500 E. Medical Center Drive/F3503,

Ann Arbor, MI 48109-0252, USA

E-mail: rjhm@umich.edu

Tel.: + 1-313-7632570

Fax: + 1-313-7649351

R. E. Ruiz

Department of Pathology,

University of Michigan Health System, Ann Arbor, MI, USA

\begin{abstract}
Background: Infantile myofibromatosis is the most common fibrous tumor of infancy. It can involve the skin, muscle, bone, and viscera. This uncommon entity is subdivided into solitary and multicentric forms, with or without visceral involvement. Objective: To describe the imaging characteristics of extracranial myofibromatosis. Materials and methods: Six infants, aged 1 day-1 week, were evaluated by imaging. All six patients had evaluation of one of the masses by US; four patients had CT evaluation of at least one of the masses; and five patients had evaluation by MRI. Results: The US appearance of the myofibromas included a mass with a purely anechoic center with a thick wall, a mass with a partially anechoic center, and a mass without anechoic components. On enhanced CT, the masses had lower or similar attenuation compared to adjacent
\end{abstract}

muscle, with some masses exhibiting peripheral enhancement. The MR appearance consisted of low signal on T1-weighted imaging. On T2-weighted imaging, two had low signal of the center and the other three had high signal. All masses showed peripheral enhancement after gadolinium administration. Conclusions: Myofibromas have variable appearance on US, with a mass with an anechoic center being the most common feature. On CT, the mass can exhibit peripheral enhancement, calcifications, and erosion of adjacent bone. The MR appearance consisted of low signal on T1-weighted imaging and high or low signal of the center on T2-weighted imaging. All masses showed peripheral enhancement after gadolinium administration.

Keywords Infantile myofibromatosis $\cdot \mathrm{CT} \cdot \mathrm{MRI} \cdot \mathrm{US}$

\section{Introduction}

Infantile myofibromatosis, the most common fibrous tumor of infancy, is a mesenchymal disorder characterized by the proliferation of fibrous tumors in the skin, muscle, bone, and viscera. Myofibromas can occur as a solitary nodule or multicentric nodules, with or without visceral involvement. Several reports describe the imaging characteristics of myofibromas [1-13]; however, description of the imaging characteristics of myofibromas other than those located in the cranium is scant. The purpose of this report is to describe the US, CT, and MRI appearance of extracranial myofibromas in six neonates.

\section{Materials and methods}

Six neonates diagnosed with extracranial myofibromatosis were evaluated by imaging. This review was exempt 
Table 1 Summary of the patients' characteristics and pathological findings

\begin{tabular}{|c|c|c|c|}
\hline Case & Age and sex & $\begin{array}{l}\text { Clinical presentation and location of } \\
\text { masses }\end{array}$ & Pathological findings \\
\hline 1 & 1-day-old boy & $\begin{array}{l}\text { Full-term born with a mass in the } \\
\text { right acromioclavicular region }\end{array}$ & $\begin{array}{l}\text { Myofibroblastic component predominant, forming thick peripheral } \\
\text { rim with micronodular appearance. Primitive component present, } \\
\text { with very prominent thin-walled vessels and abundant central } \\
\text { necrosis. Scattered zones of granular calcification within area of } \\
\text { central necrosis }\end{array}$ \\
\hline 2 & 1-day-old boy & $\begin{array}{l}\text { Full-term born with a mass in the right } \\
\text { shoulder. At } 1 \text { month of age, three } \\
\text { other masses were discovered in the } \\
\text { upper and lower back and left iliacus } \\
\text { muscle }\end{array}$ & $\begin{array}{l}\text { Myofibroblastic component predominant, forming thick peripheral } \\
\text { viable rim with increasing degenerative changes and necrosis } \\
\text { toward center of mass. Primitive component inconspicuous. } \\
\text { Necrotic portion of mass shows occasional remnants of thin-walled } \\
\text { vessels and surrounds a central cystic space. Extensive coarse } \\
\text { calcification in necrotic portion }\end{array}$ \\
\hline 3 & 1-day-old girl & $\begin{array}{l}\text { Full-term born with a mass anterior } \\
\text { to the left sternocleidomastoideus }\end{array}$ & $\begin{array}{l}\text { Myofibroblastic component forms a partial peripheral rim, which is } \\
\text { generally of low cellularity, with occasional more cellular areas. } \\
\text { Primitive component is predominant, with trabecular arrangement } \\
\text { of cells around thin-walled vessels. The primitive component shows } \\
\text { near-total necrosis. Extensive calcification present }\end{array}$ \\
\hline 5 & 1-day-old girl & $\begin{array}{l}\text { Full-term born with two masses, one in } \\
\text { the lower left posterior chest wall and } \\
\text { the other in the anterior soft tissues of } \\
\text { the right thigh }\end{array}$ & $\begin{array}{l}\text { Myofibroblastic component predominant, forming thick peripheral } \\
\text { rim. Primitive component present admixed with myofibroblastic } \\
\text { component, and as a thin viable rim between the myofibroblastic } \\
\text { component and extensive area of central necrosis. Frequent } \\
\text { remnants of thin-walled vessels and diffuse granular } \\
\text { calcification present in necrotic center }\end{array}$ \\
\hline 6 & 5-day-old boy & $\begin{array}{l}\text { Neuropathy of the left lower extremity. } \\
\text { Two masses, one in the left sciatic notch } \\
\text { and the other within the adductors of } \\
\text { the right thigh }\end{array}$ & $\begin{array}{l}\text { Small biopsies showing predominantly myofibroblastic component. } \\
\text { Primitive component limited, admixed with myofibroblastic } \\
\text { component, surrounding vessels between bundles of myofibro- } \\
\text { blasts. Extensive zones of necrosis, presumably representing center } \\
\text { of mass. Extensive coarse calcification in necrotic portions }\end{array}$ \\
\hline
\end{tabular}

from approval by the Institutional Review Board at the time it was initiated. The age at presentation, location of the masses, and pathological findings are listed in Table 1. US, CT, and MRI of the masses were performed. All six patients had evaluation of one of the masses by US; four patients had CT evaluation of at least one of the masses; and five patients had evaluation by MRI. The US evaluation, including standard grayscale and color flow Doppler, was performed with a variety of scanners. The highest-frequency transducer available was used. The CT examinations were performed after the administration of contrast media. MRI included T1-weighted spin echo, fat-suppressed T2-weighted fast spin echo, short tau inversion recovery (STIR), and T1-weighted spin echo images with fat suppression after intravenous injection of gadolinium.

\section{Results}

Table 2 summarizes the imaging findings. The US appearance of the myofibromas consisted of a mass with an anechoic center and a thick wall (1/6) (Fig. 1) and a mass with a partially anechoic center traversed by thick septa (4/6) (Fig. 2). One of the masses had an echogenic center with enhanced acoustic transmission (1/6) (Fig. 2). The masses appeared hypovascular by Doppler evaluation, though in one case there was increased flow at the periphery of the mass.

On CT after the administration of contrast media, the masses had lower (Fig. 1) or similar attenuation compared to adjacent muscle (Fig. 3), with some masses exhibiting peripheral enhancement. One of the masses had calcification within it (Fig. 3). Bone involvement was demonstrated in one case (Fig. 3).

The MR appearance consisted of low signal on T1-weighted imaging. On T2-weighted imaging, the masses had a high-signal center (3/5) (Fig. 1). In two patients, the center of the mass had low signal on T2-weighted imaging (Fig. 3). After administration of gadolinium, the myofibromas exhibited peripheral enhancement (5/5) (Figs. 1, 3).

\section{Discussion}

This report describes the imaging characteristics of myofibromatosis in six infants. The diagnosis of 


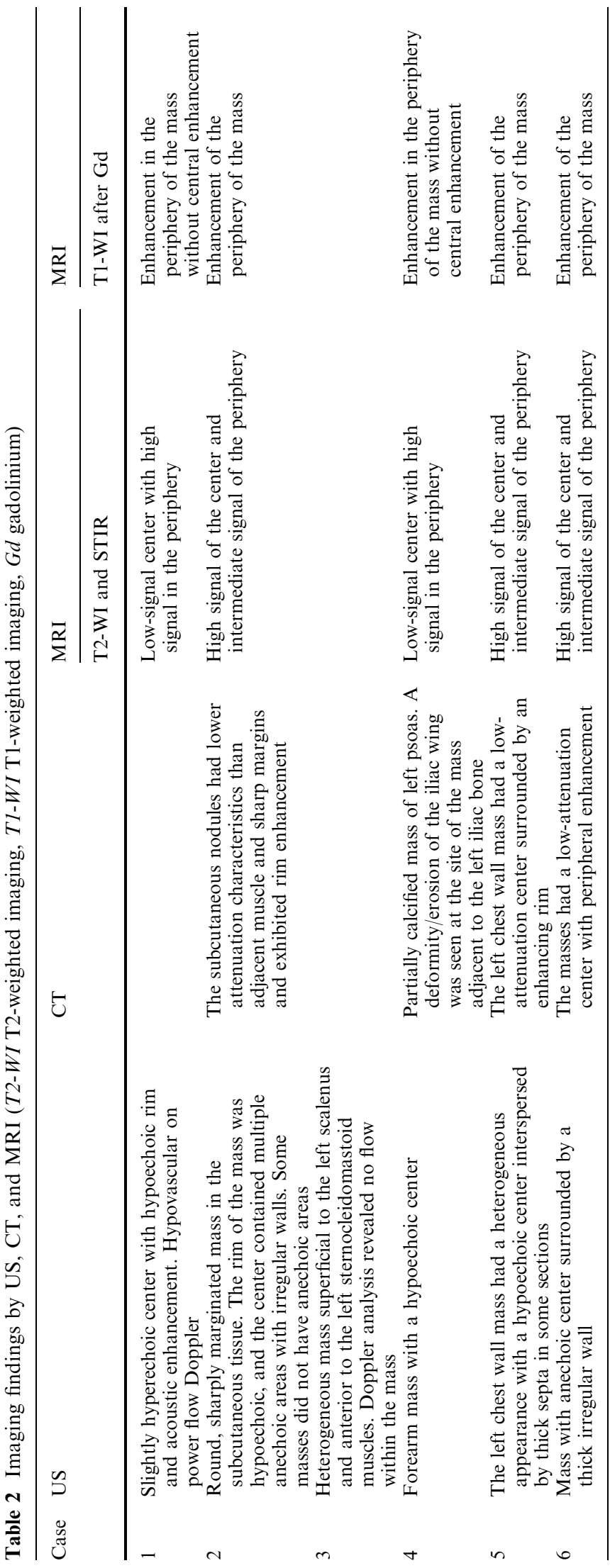

Fig. 1 A 5-day-old full-term boy (case 6) presented with left lower extremity neuropathy. a Fast spin-echo T2-weighted axial MR image $(3,500 / 83)$ of the pelvis shows ill-defined mass in the area of the sciatic notch (white arrow). The mass has intermediate signal interspersed with areas of high signal. b Axial fat-suppressed T1-weighted MR image (516/14) obtained after gadolinium administration. The mass exhibits enhancement. c Fast spin-echo T2-weighted axial MR image $(3,500 / 83)$ of the proximal thighs demonstrates a well-circumscribed mass within the adductors of the right thigh. The mass has high signal in the center with a low rim signal. d Axial fat-suppressed T1-weighted MR image (516/14) obtained after gadolinium administration. The mass shows rim enhancement with decreased signal at the center. e Axial CT image of the pelvis demonstrates a soft-tissue mass within the sciatic notch. The mass exhibits several areas of low attenuation surrounded by mild peripheral enhancement (arrow). f Axial CT image of the right thigh delineates the mass, which has lower attenuation than the muscle with irregular wall. $g$ US image of the thigh mass demonstrates a lesion with an anechoic center surrounded by a thick and irregular wall

myofibromatosis might be suggested by the presence of a single or multiple masses in a newborn or young infant, with imaging characteristics of a mass with a necrotic center. However, other findings, such as the presence of calcifications, noncystic appearance by imaging, and bone erosion, can be seen, indicating that the lesions are not uncomplicated cysts.

The current WHO classification of soft-tissue tumors uses the terms myofibroma and myofibromatosis to distinguish between solitary and multicentric processes. Although myofibromas are relatively rare overall, they are the most common fibrous tumor of infancy. They manifest as single or multiple firm, reddish-purple nodules in the skin, soft tissues, bones, and internal organs (lung, heart, gastrointestinal tract, and central nervous system). Myofibromatosis predominantly affects children younger than 2 years ( $88 \%$ of cases), but can develop later in infancy and in adults. Half of reported cases involve isolated lesions, while one-third of cases have multicentric lesions without visceral involvement. In cases without visceral involvement, the prognosis is excellent, with expected spontaneous regression of nodules in 1-2 years. Visceral lesions are associated with significant morbidity and mortality, generally within the first few months of life, secondary to obstruction of a vital organ, failure to thrive, and infection. The cardiopulmonary system and gastrointestinal tract are frequently involved. The typical histological appearance of a myofibroma is that of a nodular or multinodular neoplasm with two morphologically distinct components. One consists of bundles or fascicles of variably hyalinized myofibroblastic spindle cells with elongated vesicular nuclei. The other consists of more primitiveappearing, round to polygonal cells with hyperchromatic nuclei and scant cytoplasm, associated with thin-walled branching vessels, resembling hemangiopericytoma. The bundles of myofibroblastic cells usually form a peripheral 

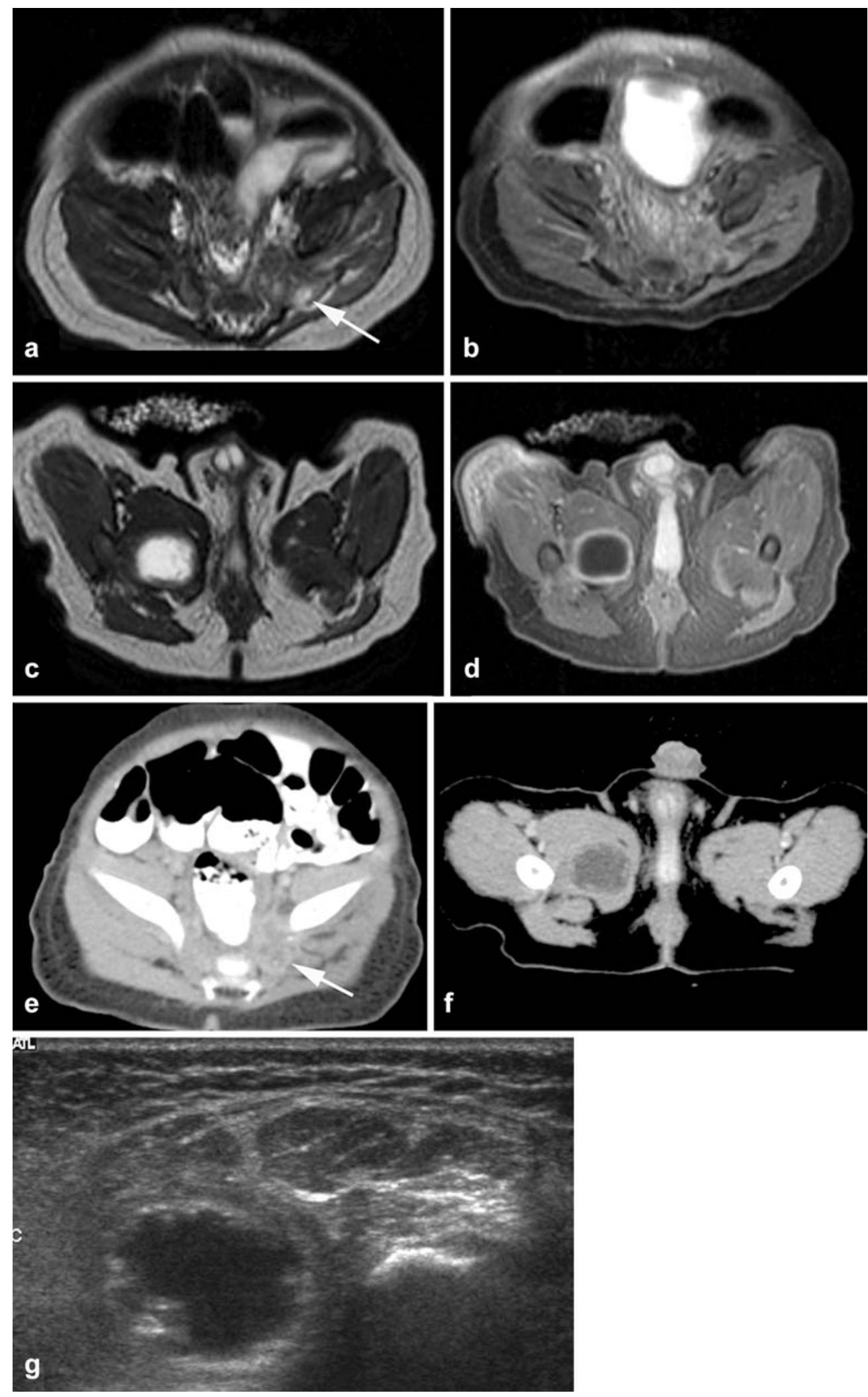
rim around a central, hemangiopericytoma-like area, the latter often showing necrosis and calcification. However, the arrangement and relative proportions of the two components vary [14].

The US appearance of myofibromas is varied. A pattern visualized in our patients was that of a mass with a predominantly anechoic center and a thick wall. The center of the mass can be traversed by thick or coarse septa. The masses did not have any flow with power Doppler except for one, which exhibited flow at the periphery of the mass. The presence of flow in some myofibromas could be a result of the vascularity demonstrated by angiography in some reports $[2,5]$.

On CT examination, masses can appear as isoattenuating with muscle or as low-attenuating with slight enhancement in the periphery. The presence of calcifications seen on CT in one of our patients has not been previously described to the best of our knowledge.

Descriptions of the MR appearance of the lesions have been varied. On T1-weighted MR images, the lesions typically appear as low-intensity foci $[15,1]$, while on T2-weighted MR images, the lesions appear as high-intensity foci $[1,9]$. A report has correlated disease regression with a collapsing peripheral rim around a very high-intensity central region on $\mathrm{T} 2$-weighted $\mathrm{MR}$ images [9]. In our series, the center of the mass on T2-weighted imaging had high signal in three patients and low signal in two patients. After the administration of gadolinium there was enhancement of the periphery, with no enhancement at the center. Schrodt and Callen [7] and Eich et al. [11] have described a similar pattern of peripheral enhancement called a "target" appearance. The imaging characteristics strongly suggest a necrotic center of these masses, consistent with the surgical and pathological findings. Central necrosis is not an uncommon pathological finding in myofibromas (Fig 4). This central necrosis might contain calcium, which could explain the low-signal center seen on T2-weighted imaging in some myofibromas.

Skeletal lesions can occur at any site, but the skull and long bones are involved most frequently. Bone involvement is common in cranio-orbito-facial bones, with the possibility of CNS involvement. Radiologically, the lesions are purely lucent, although they occasionally have a sclerotic rim.

Appropriate radiological evaluation of suspected visceral involvement in infantile myofibromatosis includes a chest radiograph, CT scan, skeletal survey, and MRI. The differential diagnosis of myofibromas involving soft tissue includes masses such as leiomyomas, neurofibromas, soft-tissue sarcomas, congenitalinfantile fibrosarcoma, hyaline fibromatosis, and deep hemangiomas, among others. Although biopsy is necessary for definitive diagnosis, age of presentation, size, imaging characteristics, and number of lesions are helpful in suggesting the diagnosis.

In conclusion, this report describes the imaging characteristics of six patients with extracranial myofibromatosis. Although the imaging characteristics of myofibromas are nonspecific, the diagnosis can be suggested when a newborn or infant presents with a mass or masses with imaging characteristics suggestive of a necrotic center. On US imaging, masses have a center that is either hyperechoic or partially anechoic and surrounded by a rim. On CT, the masses might exhibit peripheral enhancement, calcifications, and erosion of adjacent bone. On MRI, myofibromas might have a high- or low-signal center on T2-weighted imaging; however, the diagnosis of myofibromatosis is suggested by a mass with a center that has low signal and peripheral rim enhancement on T1-weighted imaging after gadolinium administration. Multiplicity of lesions as detected by clinical examination or imaging is another feature that suggests the diagnosis of multiple myofibromatosis.
Fig. 2 US images. a A 1-dayold full-term boy (case 2) with a right shoulder mass. US image demonstrates a mass with a partially necrotic center interspersed with thick septa. b A full-term boy (case 1) born with a smooth and somewhat mobile mass over the right acromioclavicular joint. US image of the soft-tissue mass superior to the humeral head (arrow). Note the slightly hyperechoic center with hypoechoic rim and acoustic enhancement
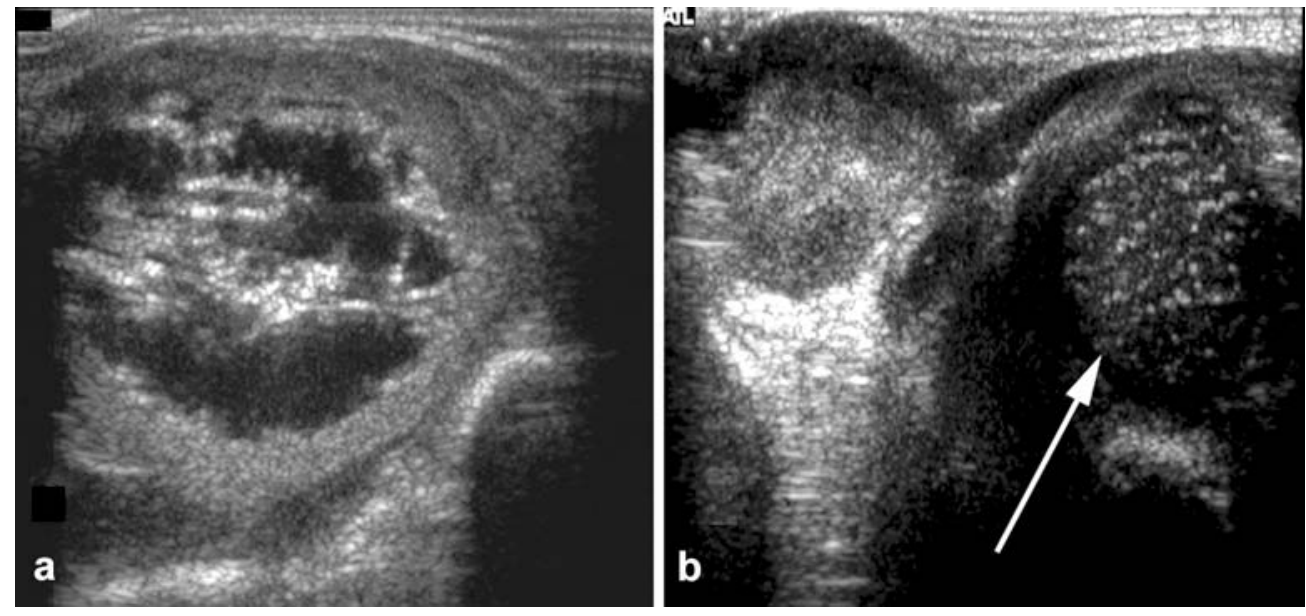

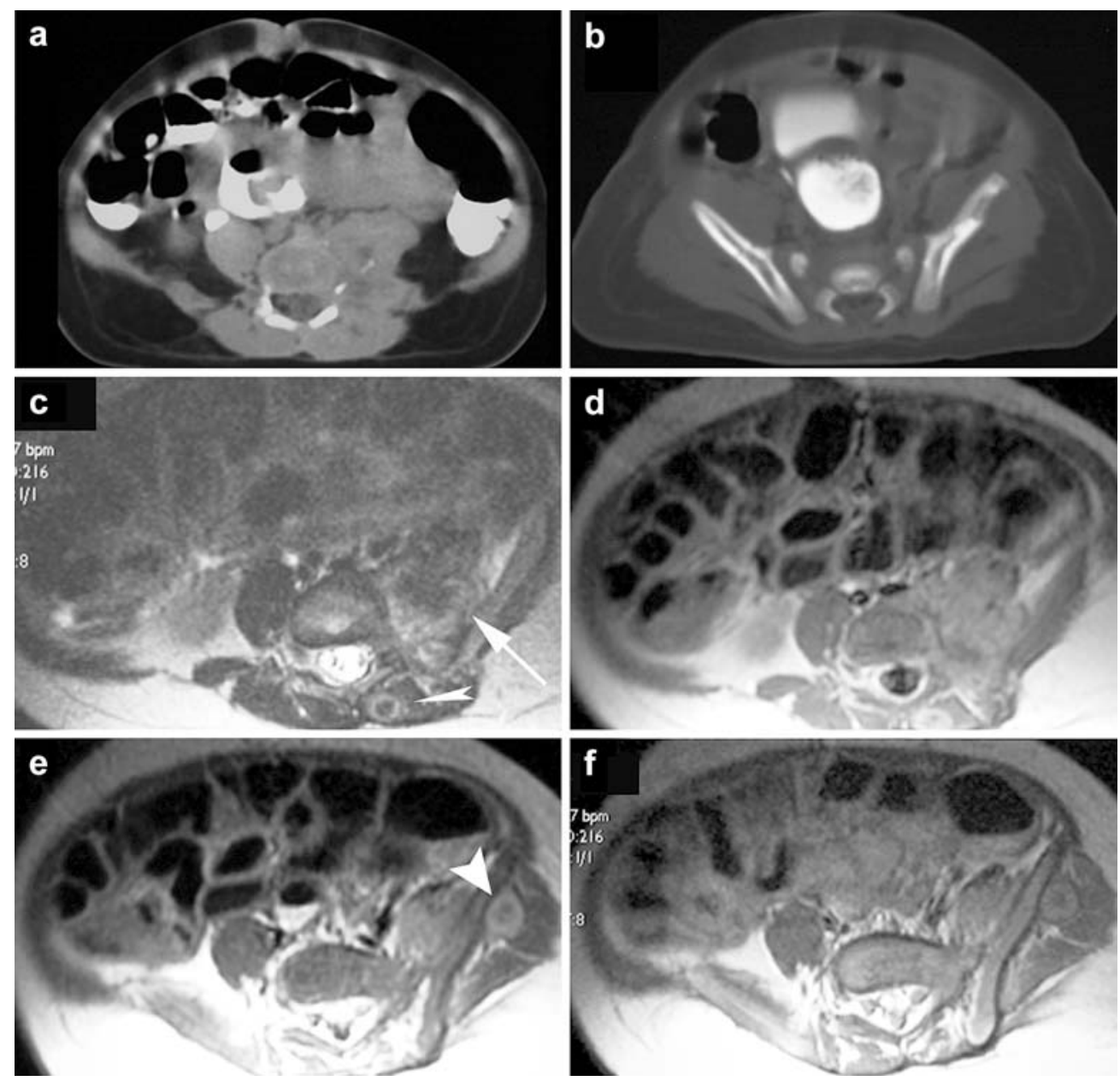

Fig. 3 A 1-week-old boy (case 4) whose parents noted decreased movement of the left leg. a Axial CT image obtained of the lower abdomen demonstrates a mass in the area of the left psoas, with intraspinal extension demonstrated on other sections. The mass exhibits several calcifications and areas of lower attenuation. A mass present in the left paraspinous musculature cannot be clearly seen. b Axial CT section through the pelvis demonstrates erosion of the left iliac bone. The soft-tissue mass adjacent to the bony deformity cannot be clearly defined. c Fast spin-echo T2-weighted $(2,835 / 115)$ MR image of the lower abdomen reveals two masses, one involving the psoas muscle (arrow) and the other involving the left paraspinous musculature (small arrowhead). Note that the centers of the masses have low signal surrounded by a rim of higher signal. d Axial T1-weighted MR image (500/11) obtained after gadolinium administration reveals minimal uniform enhancement of the mass in the psoas muscle area. The paraspinous mass has rim enhancement without enhancement of the center. e Fast spin-echo T2-weighted $(2,835 / 115)$ MR image through the pelvis demonstrates a soft-tissue mass (large arrowhead) adjacent to the left iliac bone that has similar features - a low central signal surrounded by a rim of higher signal. f Axial T1-weighted MR image (500/11) obtained after gadolinium administration shows rim enhancement with no enhancement at the center

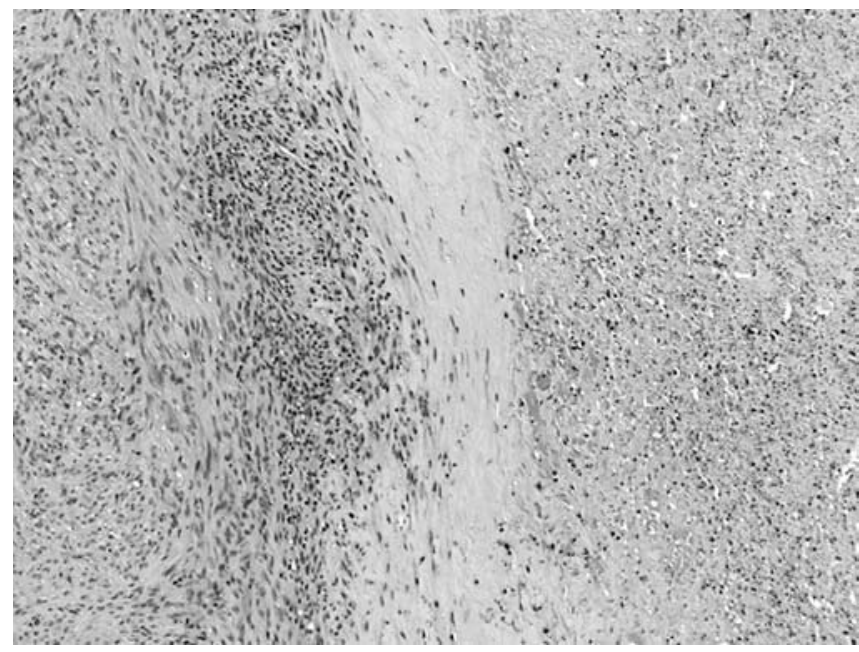

Fig. 4 Anterior right thigh mass from a patient with myofibromatosis (case 5). Myofibroblastic spindle cell component (left) forms thick peripheral rim, adjacent to and intermixed with more primitive-appearing cells with hyperchromatic nuclei (middle), surrounding the necrotic center of a mass showing diffuse granular calcification (right). H\&E, x100 


\section{References}

1. Hasegawa M, Kida S, Yamashima T, et al (1995) Multicentric infantile myofibromatosis in the cranium: case report. Neurosurgery 36:1200-1203

2. Rotigliano MJ, Pollack IF, AhdabBarmada M, et al (1994) Intracranial infantile myofibromatosis. J Neurosurg 81:539-543

3. Queralt JA, Poirier VC (1995) Solitary infantile myofibromatosis of the skull. AJNR 16:476-478

4. Netscher DT, Eladoumikdachi F, Popek EJ (2001) Infantile myofibromatosis: case report of a solitary hand lesion with emphasis on differential diagnosis and management. Ann Plast Surg 46:62-67

5. Kubota A, Imano M, Yonekura T, et al (1999) Infantile myofibromatosis of the triceps detected by prenatal sonography. J Clin Ultras 27:147-150
6. Dimson OG, Drolet BA, Southern JF, et al (2000) Congenital generalized myofibromatosis in a neonate. Arch Dermatol 136:597-600

7. Schrodt BJ, Callen JP (1999) A case of congenital myofibromatosis developing in an infant. Pediatrics 104:113-115

8. Johnson GL, Baisden BL, Fishman EK (1997) Infantile myofibromatosis. Skeletal Radiol 26:611-614

9. Moore JB, Waldenmaier N, Potchen EJ (1987) Congenital generalized fibromatosis: a new management strategy provided by magnetic resonance imaging. AJDC 141:714-716

10. Davies RS, Carty H, Pierro A (1994) Infantile myofibromatosis - a review. $\mathrm{Br}$ J Radiol 67:619-623

11. Eich GF, Hoeffel J, Tschappeler H, et al (1998) Fibrous tumors in children: imaging features of a heterogeneous group of disorders. Pediatr Radiol 28:500-509
12. Ahn J, Yoon H, Suh Y, et al (2000) Infantile fibromatosis in childhood: findings on MR imaging and pathologic correlation. Clin Radiol 55:19-24

13. Chateil J, Brun M, Lebail B, et al (1995) Infantile myofibromatosis. Skeletal Radiol 24:629-632

14. Rubin BP, Bridge JA (2002) Myofibro$\mathrm{ma} /$ myofibromatosis. In: Fletcher CDM, Unni KK, Mertens M (eds) Pathology and genetics of tumors of soft tissue and bone. IARC Press, Lyon, pp 59-61

15. Salamah MM, Hammoudi SM, Rahman A, et al (1988) Infantile myofibromatosis. J Pediatr Surg 23:975-997 